\title{
Crevisto
}

ISSN n² 2447-4266

Vol. 4, n. 2, Abril-Junho. 2018

DOI: http://dx.doi.org/10.20873/uft.2447-4266.2018v4n2p973

\section{LE MILITANT POLITIQUE A L'EPREUVE DU DIFFEREND}

THE POLITICAL MILITANT PROOF OF DIFFÉREND

O MILITANTE POLÍTICO À PROVA DO DIFFÉREND

\section{Viviane Horta Generoso ${ }^{1,2,3}$}

\section{RESUME}

Nous voulons présenter dans cette communication un exemple du concept de différend, originaire et paradigmatique, le cas de Jean-François Lyotard luimême, qu'on pourra nommer "autobiographique », jusqu'à un certain point. Il s'agit de l'épreuve intellectuelle, pratique et affective, subie lors du retrait du politique, en l'occurrence celui du marxisme, et de la perte concomitante des amis du militantisme. Lyotard s'est engagé dans le militantisme politique en 1954, quand il a pris part aux activités pratiques et théoriques du groupe qui publiait la revue Socialisme ou Barbarie. Il a milité dans ce groupe marxiste au cours de douze ans. Le militantisme était compris par tous les membres du

\footnotetext{
${ }^{1}$ Doutora em Filosofia. Pesquisadora no Laboratoire Logiques Contemporaines de la Philosophie. Département de Philosophie - Université de Paris 8. Site : http://www.llcp.univparis8.fr/spip.php?article1679. E-mail: hortaviviane@gmail.com.

${ }^{2}$ Texte présenté lors du $4^{\mathrm{e}}$ colloque "Politique de la Philosophie, philosophie de la politique. Réflexions autour de langages partagés », le 9 décembre 2015. Organisé par le Département de Philosophie de Paris 8 et le Secrétariat de la Recherche et de la Faculté de Sciences Politiques et Relations Internationales de l'Université Nationale de Rosario (Argentine), sous la direction de M. Patrice Vermeren et Mme. Angélica Montes Montoya. Travail qui a été financé par la Fondation Capes (Subordonnée au Ministère de l'Éducation du Brésil - Doutorado Pleno no Exterior, $n^{\circ}$ : BEX 1067/14-1).

${ }^{3}$ Adresse de contact avec l'auteur (par courrier) : 5, Résidence du Parc de la Faisanderie, 95290 - L'Isle Adam - France.
} 


\section{Grevisto}

ISSN n² 2447-4266

Vol. 4, n. 2, Abril-Junho. 2018

DOI: http://dx.doi.org/10.20873/uft.2447-4266.2018v4n2p973

groupe comme une exigence d'engagement existentiel. Cet engagement existentiel exigeait une manière de vivre spécifique. Cela veut dire que le sens d'une vie d'activiste politique est indissociable de sa manière de penser et d'agir dans la guerre entre exploiteurs et exploités. Mais, un évènement surviendrait qui fera une fissure au cœur de cet engagement. Le tournant s'ouvrira lors qu'un sentiment de soupçon sur la légitimation du discours marxiste se signalera. Ce sentiment ouvre une blessure dans le militant, au fur et à mesure qui commençait à suspecter " la validité du marxisme à exprimer les changements $d u$ monde contemporain». Ce sentiment signale qu'il y a là «quelque chose » et c'est précisément la recherche de ce «quelque chose» que va travailler le militant, c'est-à-dire, exigera de lui un nouveau labeur de transformation de soi. Ce qui nous intéresse ici spécialement, c'est la manière dont Lyotard travaille cette blessure de la légitimation et la perte de l'amitié des camarades de lutte et du militantisme; pertes irrémissibles qui l'obligeront à reprendre à nouveau frais le principe orientant sa manière de penser et de vivre.

MOTS-CLÉS: Militant; Lyotard; differend; Philosophie.

\section{ABSTRACT}

This paper presents an example of the concept of différend, original and paradigmatic: the case of Jean-François Lyotard himself, which we can call "autobiographical", to a certain extent. This case reports the intellectual, practical and affective challenge that Lyotard suffered from his withdrawal from political militancy, in this case Marxism, and the concomitant loss of friends of militancy. Lyotard engaged in political activism in 1954, when he took part in the practical and theoretical activities of the group that published in France the journal Socialisme ou Barbarie. He has been a member of this Marxist group for twelve years. Political militancy was seen by the group as a demand for existential engagement. This existential engagement required a specific way of living for all. That is to say, that the meaning of a life of political activist was inseparable from his way of thinking and acting in the war between the exploiters and the exploited. But the occurrence of an unexpected event will cause a fissure at the center of this existential engagement. This change will 


\section{Grevisto}

ISSN n² 2447-4266

Vol. 4, n. 2, Abril-Junho. 2018

DOI: http://dx.doi.org/10.20873/uft.2447-4266.2018v4n2p973

come about because of a sense of suspicion about the legitimacy of Marxist discourse. This feeling opens a wound in the militant, the extent to which he begins to suspect the "validity of the Marxist discourse in understanding the changes of the contemporary world." This feeling point out that there is "something" which he will name a posteriori différend, that is: a kind of contradiction or conflict that is irreducible to the dialectic, even Marxist. And it is precisely the search for this "something" that will work the militant, that is, it will require a new transformation of itself. What interests us here in particular is the way in which Lyotard works this fissure, this pain of the loss of legitimation and consequently of the friendship of the militant comrades. Unforgivable (in the eyes of fighting friends) and irreparable loss, hat will force him to put in new terms the principle that guides his way of thinking and living.

KEYWORDS: Activist; Lyotard; différend, Philosophy.

\section{RESUMO}

Esse texto apresenta um exemplo do conceito de différend, original e pragmático:o caso de Jean-François Lyotard, que podemos nomear como sendo "autobiográfico", até um certo ponto. Este caso relata o desafio intelectual, prático e afetivo, que Lyotard sofreu com o a sua saída da militância política, no caso o marxismo, e a perda concomitante dos amigos de militância. Lyotard se engajou na militância política em 1954, quando participou das atividades práticas e teóricas do grupo que publicava na França a Revista Socialismo ou Barbárie. Ele militou neste grupo marxista cerca de doze anos. A militância política era vista pelo grupo como uma exigência de engajamento existencial. Esse engajamento existencial reclamava de todos uma maneira de viver específica. Isso quer dizer, que o sentido de uma vida de ativista político era indissociável da sua maneira de pensar e de agir na guerra entre os exploradores e os explorados. Mas a ocorrência de um evento inesperado causará uma fissura no centro deste engajamento existencial. Essa mudança acontecerá devido a um sentimento de suspeita sobre a legitimação do discurso marxista. Esse sentimento abre uma ferida no militante, à medida em que ele começa a suspeitar da "validade do discurso marxista em 


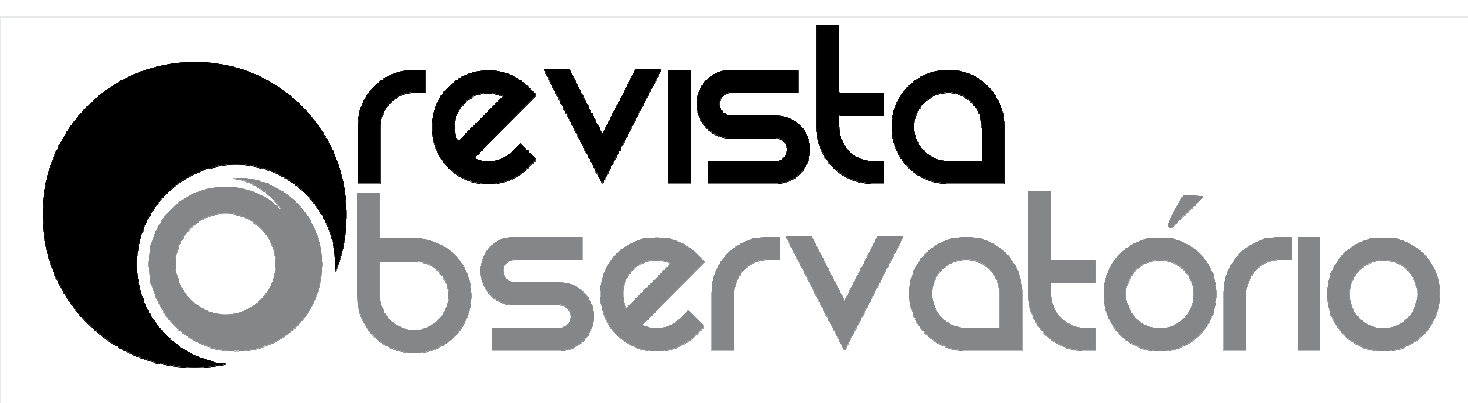

ISSN n² 2447-4266

Vol. 4, n. 2, Abril-Junho. 2018

DOI: http://dx.doi.org/10.20873/uft.2447-4266.2018v4n2p973

compreender as mudanças do mundo contemporâneo". Esse sentimento assinala que existe "alguma coisa" que ele nomeará a posteriori différend, ou seja : um tipo de contradição ou de conflito que é irredutível à dialética, mesmo marxista. E é precisamente a busca dessa "alguma coisa" que vai trabalhar o militante, ou seja, exigirá dele uma nova transformação de si. O que nos interessa aqui especialmente, é a maneira pela qual Lyotard trabalha esta fissura, essa dor da perda de legitimação e consequentemente da amizade dos camaradas de militância. Perda imperdoável (aos olhos dos amigos de luta) e irreparável, que o obrigará a colocar em novos termos o principio que orienta a sua maneira de pensar e de viver.

PALAVRAS-CHAVE: Ativista; Lyotard; différend; Filosofia.

Recebido em: 30.10.2017. Aceito em: 16.12.2017. Publicado em: 01.04.2018. 


\section{Grevisto}

ISSN n² 2447-4266

Vol. 4, n. 2, Abril-Junho. 2018

DOI: http://dx.doi.org/10.20873/uft.2447-4266.2018v4n2p973

Je voudrais présenter dans cette communication un exemple originaire et paradigmatique du concept de différend.

Il s'agit du cas de celui même dont le nom reste attaché à la production de ce concept : Jean-François Lyotard.

En quoi on pourra déceler dans son livre de philosophie par excellence, Le Différend, une dimension sous-jacente et pour ainsi dire « autobiographique ».

Par différend j'entends ici l'épreuve intellectuelle, pratique et affective, subie par Lyotard militant lors de la perte ou du retrait du politique, en l'occurrence celui du marxisme.

À vrai dire, c'est à l'issue de cette épreuve que le terme de différend viendra nommer et donner à penser ce qui se sera joué dans l'expérience vécue difficilement communicable de cette perte.

Je vais procéder ici en trois parties: la première partie concerne au militant ; la deuxième c'est sur la « genèse affectuelle » du concept de différend chez Lyotard et la troisième, est l'événement de la rupture du militant avec le marxisme et la « naissance » du concept de différend.

\section{I}

\section{Le militant}

Lyotard s'est engagé dans le militantisme politique en 1954, quand il a pris part aux activités pratiques et théoriques du groupe qui publiait la revue Socialisme ou Barbarie. Ce groupe militant fondé, dans les années 1948, par Cornelius Castoriadis et Claude Lefort, avait osé tourner les armes de la critique 


\section{Grevisto}

ISSN n² 2447-4266

Vol. 4, n. 2, Abril-Junho. 2018

DOI: http://dx.doi.org/10.20873/uft.2447-4266.2018v4n2p973

radicale vers ce qui paraissait intouchable à cette critique même, à savoir le discours marxiste et ledit communisme «réel ». Cette audace était d'une importance décisive pour l'engagement de Pierre Souyri et de Lyotard au groupe marxiste. Et ce qui paraissait particulièrement intouchable à la critique était de s'éloigner des positions trotskistes, au sortir de la Seconde Guerre mondiale. "Dans cette radicalité retrouvée, il y avait un cri de délivrance (...) il fallait d'abord (...) constater l'incapacité du trotskisme de mener à bien sa critique théorique», remémore Lyotard dans son texte "Mémorial pour un marxisme : À Pierre Souyri ». ${ }^{4}$

Lyotard a milité dans ce groupe marxiste au cours de douze ans. Pendant cette période, "il a dédié totalement son temps et sa capacité de penser et d'agir à la seule entreprise critique et d'orientation révolutionnaire» (MPM, p. 95). Le militantisme était compris par tous les membres du groupe comme une exigence d'engagement existentiel. Ils avaient l'habitude de se réunir tout le temps et autant qu'il fallait pour débattre sur les questions politiques; comme Lyotard le dit lui-même: "Rien d'autre, à part aimer, ne nous avait paru valoir un instant de distraction pendant ces années » (MPM, p. 96).

Cet engagement existentiel exigeait une manière de vivre spécifique. Cela veut dire que le sens d'une vie d'activiste politique est indissociable de sa manière de penser et d'agir dans la guerre entre exploiteurs et exploités. II n'y a pas de tolérance pour l'esprit qui oublie le but du militantisme radical: la destruction de l'exploitation par la pensée et par les actes. Même les amis le plus chers ne bénéficiaient d'aucun privilège dans la relation militante; tout le

\footnotetext{
${ }^{4} \mathrm{Cf}$, «Pierre Souyri : le marxisme qui n'a pas fini », Introduction à : P. Souyri, Révolution et contrerévolution en Chine, C. Bourgois, 1982; repris dans J.-F. Lyotard, Pérégrinations, Galilée, 1990, sous le titre de "Mémorial pour un marxisme : à Pierre Souyri », p.118. Dorénavant désigné par MPM.
} 


\section{Grevisto}

ISSN n² 2447-4266

Vol. 4, n. 2, Abril-Junho. 2018

DOI: http://dx.doi.org/10.20873/uft.2447-4266.2018v4n2p973

monde devait argumenter sa réfutation du système par raison et par preuves. Le marxisme critique, dit Lyotard, disposait d'un appareil de lecture des faits (jamais établis), mais toujours possibles d'arbitrer par principe entre ce qui mérite et ce qui ne mérite pas attention dans la lutte des idées, entre ce qui continue à vouloir l'émancipation concrète des exploités comme son but et ce qui cesse de le vouloir.

Il serait aisé de montrer que cette nécessité d'engagement existentiel dans le politique demande une transformation de soi et une conversion de soi vers une nouvelle manière de vivre et de penser. Conversion qui modifie le sujet en l'emmenant d'une vie qu'on pourrait appeler «non authentique » (reçue, déjà donnée) vers une vie plus authentique (la nouvelle vie, vita nuova, ou « vraie vie ») dans laquelle il prend conscience (politique) de soi et du monde. ${ }^{5}$

\footnotetext{
${ }^{5}$ Pierre Hadot rappelle que le mot conversio correspond en grec à deux mots : " epistrophè et metanoia » "Epistrophè signifie changement d'orientation et implique l'idée d'un retour (retour à l'origine, retour a soi); metanoia signifie changement de pensée, repentir, et implique l'idée d'une mutation et d'une renaissance ». Il existe donc, dans le concept, une opposition d'idées: la première serait "le retour à l'origine », la seconde "la renaissance »; cf. Hadot, l'Encyclopaedia Universalis, mot: conversion; disponible en ligne: www.universalis.fr. Nous verrons que Michel Foucault problématisera cette opposition en introduisent une troisième catégorie. Nous essayerons par la suite de situer la conversion militante par rapport à ceux trois catégories.

Hadot souligne que les philosophes dans les écoles épicuriennes, stoïciennes et néoplatoniciennes étaient préoccupées par la conversion des âmes humaines. La conversion était un événement provoqué dans l'âme du récepteur par le moyen des mots et des gestes d'un philosophe. Ce type de conversion «correspond à une rupture totale avec la manière habituelle de vivre: changement de costume, et souvent de régime alimentaire, parfois renonciation aux affaires politiques, mais surtout transformation totale de la vie morale, pratique assidue de nombreux exercices spirituels ». (cf. Pierre Hadot, Exercices spirituels et philosophie antique, Albin Michel, p. 226).

À la différence de la conception de P. Hadot sur la conversion, commandée par l'opposition de deux acceptions du terme, epistrophè et metanoia, Foucault développe donc un troisième aspect de l'idée de conversion. Cette troisième modulation nuancée serait celle de la conversion hellénistique de soi, que figure la conversion du regard. Foucault souligne que lorsque Plutarque, Epictète, Sénèque, Marc Aurèle, par exemple, ont affirmé l'importance de " se regarder soi-même ", ce regard, qui se distingue du "regard vers soi-même ", a un sens distinct du « connais-toi toi-même » platonicien, ou encore du « examine-toi toi-même » de la
} 


\section{arevisto}

ISSN n² 2447-4266

Vol. 4, n. 2, Abril-Junho. 2018

DOI: http://dx.doi.org/10.20873/uft.2447-4266.2018v4n2p973

La "vraie vie », dans le cas du militant, résidait dans la lutte des classes et dans l'effort pour faire disparaitre les rapports d'exploitation. Le marxisme avait été la seule manière convaincante de répondre au défi lancé par le capitalisme à la liberté et au sens de l'histoire en faisant resurgir le conflit là où il était étouffé, dit clairement Lyotard. Nous pouvons dire que le marxisme était, pour lui et pour les militants du groupe Socialisme ou Barbarie, comme une "chance à saisir », celle d'une thérapeutique apte à entendre l'inconscient de l'histoire.

Il fallait écouter l'inconscient de l'histoire, l'expérience de luttes, comme on prête l'oreille au patient, mais aussi défendre cette expérience contre ce qui en elle-même travaillait à la défigurer (MPM, p.128).

L'exploitation était un nom de « la chose » irréfutable de la vie.

Elle seule méritait la dépense sans mesure de toute intelligence et de toute volonté. On ne pouvait se réconcilier avec soi-même, être heureux et intelligent, jouir de la vie, tant qu'elle était là. Elle était le malheur, la maladie et la promesse de la mort pesant sur l'espèce. Et pas la "belle mort" qui sauve l'esprit, mais la misère qui l'exténue, le condamne à la répétition, l'abuse et l'élimine. (Nous qui soulignons, MPM, p. 127.)

Nous pouvons sentir, dans ce remarquable témoignage de Lyotard, les exigences transformatrices de soi que demande la vie militante: la recherche

spiritualité monastique. Foucault établit ainsi une distinction entre la conversion du regard platonicien l'épistrophè (exercice de la connaissance), la conversion hellénistique et romaine, la conversio (exercice de concentration) et la conversion chrétienne, la metanoia (exercices de déchiffrement). Pour Platon, l'orientation du regard a pour objectif de convertir à soi-même comme objet de connaissance; dans le christianisme, il se présente comme surveillance des images et des représentations qui peuvent envahir et noircir notre âme; dans les sens hellénistique et romain, il s'agit plutôt de détourner le regard des autres et du monde extérieur. Pour le tourner vers soi-même (Cf. Foucault, L'Herméneutique du Sujet, p 208). 


\section{Grevisto}

ISSN n² 2447-4266

Vol. 4, n. 2, Abril-Junho. 2018

DOI: http://dx.doi.org/10.20873/uft.2447-4266.2018v4n2p973

incessante des militants pour supprimer l'exploitation, afin de donner un sens, une orientation à leur vie et à la vie.

Or, cette exigence de transformation, nous la retrouvons comme les éléments et exigences de la spiritualité, dirait Michel Foucault, ou des exercices spirituels, dirait Pierre Hadot, pour pouvoir mener une vraie vie. Nous savons par ailleurs que Foucault, à la fin de sa vie, a examiné l'activité révolutionnaire, non seulement comme un projet politique, mais aussi comme une forme de vie. En effet, le militantisme du XIX et du XX $X^{\mathrm{e}}$ siècles en Europe a compris la vie révolutionnaire selon trois aspects : la socialité secrète; l'organisation instituée ; et le témoignage par la vraie vie, la vie comme style d'existence. Foucault fait alors remarquer que

ce style d'existence propre au militantisme révolutionnaire, et assurant le témoignage par la vie, est en rupture, doit être en rupture avec les conventions, les habitudes, les valeurs de la société. Et il doit manifester directement, par sa forme visible, par sa pratique constante et son existence immédiate, la possibilité concrète et la valeur évidente d'une autre vie, une autre vie qui est la vraie vie. (...) $\mathrm{Ce}$ thème, si fondamental et en même temps si énigmatique, et si intéressent, de la vraie vie, cette vraie vie dont le problème a été posé par Socrate déjà et dont la thématique n'a pas cessé, je crois, de parcourir toute la [pensée] occidentale. ${ }^{6}$

Deux années avant le cours sur Le courage de la vérité, Foucault a esquissé dans le cours sur l'Herméneutique du sujet, la différence entre les formes de savoir qui relève de la connaissance et celles que ne sont pas des sciences et où nous retrouvons certaines exigences de la spiritualité. Les

\footnotetext{
${ }^{6}$ Foucault, Le courage de la vérité, leçon du 26 février 1984, Gallimard, p. 170.
} 


\title{
Crevisto
}

ISSN n² $2447-4266$

Vol. 4, n. 2, Abril-Junho. 2018

DOI: http://dx.doi.org/10.20873/uft.2447-4266.2018v4n2p973

exemples qu'il en donne sont justement ceux du marxisme et de la psychanalyse.

Dans le marxisme comme dans la psychanalyse, le problème de ce qu'il en est de l'être du sujet (de ce que doit être l'être du sujet pour qu'il ait accès à la vérité ) et la question en retour de ce qui peut se transformer du sujet du fait qu'il a accès à la vérité, eh bien ces deux questions, qui sont encore une fois des questions absolument caractéristiques de la spiritualité, vous les retrouvez au cœur même ou, en tout cas, au principe et à l'aboutissement de l'un et de l'autre de ces savoirs ${ }^{7}$.

Nous avons là, au passage une précision sur ce qui spiritualité veut dire, à savoir, le exercice que le sujet doit faire sur lui même pour se transformer et se rendre apte à la vérité. Notons aussi que Foucault n'identifie pas cette forme de savoir que sont le marxisme et la psychanalyse aux formes de spiritualités; il repère seulement, dans le marxisme et dans la psychanalyse des éléments concernant le travail sur soi que sont caractéristique de la spiritualité.

\begin{abstract}
Je ne dis pas du tout que sont des formes de spiritualités. Je veux dire que vous retrouvez dans ces formes de savoir les questions, les interrogations, les exigences qui, me semble-t-il - à prendre un regard historique sur quelques millénaires, au moins sur un ou deux -, sont les très vieilles, les très fondamentales questions de l'epimeleia heautou, et donc de la spiritualité comme condition d'accès à la vérité. (Ibid.)
\end{abstract}

Si le marxisme est donc une forme spécifique de savoir, parente de la spiritualité, dans le sens esquissé par Foucault, alors le militant ne peut être en effet un véritable marxiste que s'il fait un travail de conversion sur lui-même. Une conversion de son mode être et tout d'abord un changement de sa

\footnotetext{
${ }^{7}$ Foucault, L'Herméneutique du sujet, leçon du 6 janvier 1982, Gallimard, p. 30 et 31.
} 


\section{Crevisto}

ISSN n² 2447-4266

Vol. 4, n. 2, Abril-Junho. 2018

DOI: http://dx.doi.org/10.20873/uft.2447-4266.2018v4n2p973

manière de penser et d'agir. Or, tout cela s'accorde parfaitement avec les descriptions de Lyotard, en parlant de son propre exemple et de son engagement existentiel dans la lutte des classes pendant les douze années de Socialisme ou Barbarie.

Mais, un évènement surviendrait qui fera une fissure au cœur de cet engagement. Le tournant s'ouvrira lors qu'un sentiment de soupçon sur la légitimation du discours marxiste se signalera. Ce sentiment ouvre une blessure dans le militant, au fur et à mesure qui commençait à suspecter "la validité $d u$ marxisme à exprimer les changements du monde contemporain » (MPM, p. 98).

Ce sentiment signale qu'il y a là « quelque chose » et c'est précisément la recherche de ce "quelque chose » que va travailler le militant, c'est-à-dire, exigera de lui un nouveau labeur de transformation de soi. Ce qui nous intéresse ici spécialement, c'est la manière dont Lyotard travaille cette blessure de la légitimation et la perte de l'amitié des camarades de lutte et du militantisme; pertes irrémissibles qui l'obligera à reprendre à nouveau frais le principe orientant sa manière de penser et de vivre.

II

\section{Soupçon et « genèse affectuelle » du concept de différend}

Le marxisme était considéré par les militants un langage à valeur universelle. C'était une manière de réfléchir sur la réalité, où la dialectique du discours incorporait par définition tout ce qui était censé contredire celui-ci.

Autrement dit, 


\section{Grevisto}

ISSN n² 2447-4266

Vol. 4, n. 2, Abril-Junho. 2018

DOI: http://dx.doi.org/10.20873/uft.2447-4266.2018v4n2p973

un langage à valeur universelle, capable même d'accueillir en lui, sous le nom de logique dialectique, la rupture et l'opposition d'universaux qui étaient des abstractions, et le mouvement paradoxal et infini par lequel ils se réalisent concrètement. (MPM, p. 98 et 99$)^{8}$.

Dès que Lyotard à commencer a éprouvé un sentiment de soupçon à l'égard du discours marxiste comme langage à la valeur universelle, il a pressentir aussi que cette logique dialectique, avec son opérateur irréfutable et l'anti-principe de contradiction, était en train de devenir un idiome simplement particulier, comme un autre. En ce sens, tous les débats entre les militants été nécessairement concentré sur la « langue marxiste ».

La question nouvelle qui apparaît alors au militant Lyotard est de savoir comment parler et débattre, au-delà des contenus de la langue marxiste (le capitalisme moderne, la baisse du taux de profit, le prolétariat, etc.), pour débattre de cette langue elle-même. Et toute la difficulté résidera dans le faite que cette langue paraitrait interdire qu'on puisse la prendre elle-même pour objet de discussion.

Il me semblait, écrit-il, que le discours qui se nomme matérialisme historique faisait parler son référent, la réalité historique, dans le langage de la lutte des classes. Or celui-ci était un genre de discours, et il avait ses règles, comme de juste, mais ses règles m'interdisaient précisément de le traiter comme un genre, parce qu'il prétendait pouvoir les transcrire tous ou, ce qui revient au même, pouvoir tout dire de son référent.(MPM, p. 103).

\footnotetext{
${ }^{8}$ Nous allons revenir plus tard sur la question du rapport entre la dialectique et le différend. Voir J-F. Lyotard «Essai d'analyse du dispositif spéculatif », Degrés, n 26-27 (1991); repris dans Lyotard à Nanterre, Klincksieck, 2010. Cette question est au cœur de la querelle philosophique franco-allemande (entre Habermas et Lyotard). Comme la montre P. Prado, "Le sentiment du différend, ou : la critique de la raison communicationnelle (Lyotard lecteur de Wittgenstein) ». In Passages de J.-F. Lyotard, P. W. Prado, coll. J. Poulain et B. Cany, Hermann, Paris 2011.
} 


\section{Qrevisto}

ISSN n² 2447-4266

Vol. 4, n. 2, Abril-Junho. 2018

DOI: http://dx.doi.org/10.20873/uft.2447-4266.2018v4n2p973

En somme ce soupçon que le matérialisme dialectique était aussi un genre de discours particulier, avec ses propres règles, n'avait pas le moins de s'exprimer - dans le cadre de ce genre de discours. Autrement dit que la prétention du marxisme à être un discours universel, qui croit pouvoir exprimer sans résidu toutes les positions antagonistes, sa prétention à être un métalangage qui voudrait tout dire de son référent, cela rendrait inaudible par avance tout soupçon. Tout cela fait qu'à partir de ce moment Lyotard ne peut plus continuer à parler cette langue; de là vient la difficulté de parler et de discuter aussi avec ses camarades, en particulier avec son ami Pierre Souyri.

Cette difficulté relevait d'un problème de logique. Comme il n'y a plus une langue commune, les camarades ne peuvent plus s'expliquer et ni même exprimer leur désaccord. Différemment d'une simple divergence, Lyotard se trouve devant un cas particulier qu'il va nommera par la suite un différend. Dans ce cas, l'objet en question (la langue marxiste) ne peut pas être thématisé dans le débat, à moins d'en modifier les règles du discours (celles mêmes de la langue marxiste). Comme il le remarque lui-même dans son Mémorial dédié à Souyri :

cette incommensurabilité n'était pas symétrique, mais déséquilibrée. L'un des idiomes [le marxisme] s'offrait à dire ce qui était la situation «même », à expliquer en quoi il s'agissait bien du «même » référent d'un côté et d'autre, donc à se présenter non pas comme une partie dans un procès, mais comme le juge, comme la science détentrice de l'objectivité, plaçant ainsi l'autre dans la position de stupeur ou de stupidité que j'avais connue, le confinant dans la particularité subjective d'un point de vue qui restait incapable de se faire comprendre, sauf à emprunter l'idiome dominant, c'est-à-dire à se trahir. (MPM, p. 115, 116) 


\section{arevisto}

ISSN n² 2447-4266

Vol. 4, n. 2, Abril-Junho. 2018

DOI: http://dx.doi.org/10.20873/uft.2447-4266.2018v4n2p973

Cette dissymétrie entre la nouvelle situation de Lyotard (se déprenant du discours marxiste) et celle de Pierre Souyri (parlant toujours ce discours) au sujet du discours marxiste lui-même a fait éclater entre eux ce que serait nommé plus tard un différend. Mais même dans cette situation défavorable, Lyotard cherche à trouver les mots, à trouver un idiome, qui soit capable d'articuler ce sentiment de stupeur, de stupidité qui fait qu'il ne peut pas se faire comprendre de l'autre (et à la limite, même pas de lui-même); de son côté, l'autre est devenue partie et juge dans le procès.

Or, cela qu'il nous raconte et qu'il nous fait ressentir remarquablement dans son témoignage à la mémoire de son ami Souyri, c'est précisément une épreuve, un sentiment du différend, avant la lettre, dont l'écoute aura précédée et présidé à la création du concept de différend. Après éprouver ce sentiment, la rupture avec l'ami et le militantisme était inévitable.

III

\section{L'événement de la rupture}

L'événement de la rupture a lieu pour Lyotard après l'occasion de la scission du groupe Socialisme ou Barbarie en 1964, laquelle accomplit le divorce entre une «tendance» animée par Cornelius Castoriadis, qui veut poursuivre la publication de la revue Socialisme ou Barbarie, et l'autre courant, avec Pierre Souyri, qui se consacrait à publier le mensuel Pouvoir ouvrier. ${ }^{9}$ Mais

\footnotetext{
${ }^{9}$ Lors de cette scission du groupe en 1964 Lyotard se trouvait du côté du groupe de Souyri. La nouvelle «tendance » animée par Castoriadis était quelque chose de suspect pour Lyotard,
} 


\section{Qrevisto}

ISSN n² 2447-4266

Vol. 4, n. 2, Abril-Junho. 2018

DOI: http://dx.doi.org/10.20873/uft.2447-4266.2018v4n2p973

le tournant s'effectuera pour lui deux ans après, en 1966, l'année où Lyotard démissionne du groupe "Pouvoir ouvrier ». De sont propre aveu, cela lui a été une douloureuse rupture et avec le groupe et avec ses camarades de lutte. "Une période de ma vie finissait, je quittais le service de la révolution. » (MPM p. 96).

La scission d'avec le militantisme radical était l'accomplissement de l'événement qui avait frappé le militant Lyotard. Cet événement était signalé par un sentiment de différend entre quelque chose qui voudrait se dire (s'articuler), et l'impossibilité de le faire pour l'instant. Lyotard décrit magnifiquement tous les sentiments que l'ont affecté au cours du processus de rupture, ceux d'être méprisé, d'inhibition, de colère, de stupeur, d'impuissance. Tout cela qui l'empêchait d'articuler qui lui lassait stupide, comme il le dit lui-même : "Je me sentais méprisé pour la direction que j'avais prise, comme je savais que l'avaient été par nous les intellectuels et les politiques retirés du combat de classe ou aveugles à son enjeu. »

Et Lyotard d'ajouter :

De sorte que ce différend prit un tour paradoxal. Il me remplissait de colère, mais aussi me lassait stupide. Je me trouvais sans mots pour dire, pour me dire, ce que pouvait signifier et valoir l'attachement de Souyri au monde de pensée marxiste. Bien plus : je pouvais encore à sa place et dans son genre de discours accabler ma propre irrésolution, je ne voyais pas comment, dans quel genre, à quelle place, qui auraient dû être les miens, m'en prendre à ses certitudes à lui. Il me sembla de façon obscure et inintelligente qu'il ne fallait pas

malgré son accord concernant le contenu des thèses avancées. " /l y avait quelque chose qui ne se laissait pas corrompe par les trésors d'argumentation que la tendance, et notamment Castoriadis, dépensaient pour expliquer et justifier la nouvelle orientation. II ne manquait rien à la panoplie argumentative de ces camarades, et pourtant cette saturation révélait un manque. (...) Je parle du ton et de la méthode, car quant au contenu, il était plutôt existentiel. On faisait la toilette du marxisme, on lui passait des habits neufs. La vieille contradiction du Capital, jugée économiste, était mise à la poubelle. »Cf. MPM, p.113 et 114. 


\section{grevisto}

ISSN n² 2447-4266

Vol. 4, n. 2, Abril-Junho. 2018

DOI: http://dx.doi.org/10.20873/uft.2447-4266.2018v4n2p973

me hâter de surmonter cette dissymétrie ni de rétablir l'incompréhension à parité.

Ce ne devait être qu'à force de ne pas faire mon deuil de mon impuissance que pouvait s'esquisser, pensais-je sans raison, une autre manière de penser, comme en mer le nageur incapable de riposter au courant fait confiance à la dérive pour trouver un autre abord. ${ }^{10}$

Il y a eu un retrait d'investissement affectif à l'égard du mouvement marxiste, et en sens à l'essuie du militant. Ce retrait d'investissement affectif attestait un retrait de la crédibilité de la langue du radicalisme marxiste.

Dans les deux passages que vient d'être cités, nous prenons la mesure de la difficulté de ce geste de rupture; nous pouvons même "sentir » la souffrance dans les mots chargés de sentiments que sont utilisé. Mais, en même temps, nous comprenons que la rupture était sans remède; ce faisant elle tantôt inaugurerait la dérive qui lui écarterait de Souyri et du «service de la révolution » tantôt ouvrira la voie à la production du concept de différend.

Qu'est qui se passe quand le militant n'adhère plus au discours marxiste? N'est faut-il pas s'engager dans un nouveau travail sur soi, dans une

\footnotetext{
${ }^{10}$ Cf . MPM, p. 97 et p.105. (Nous qui soulignons). Est-il besoin de souligner l'importance du mot dérive dans l'œuvre de Lyotard? Lui même défini le mot comme suit: "Derivatio n'est pas du tout quitter une rive, mais détourner un rivus, un cours, une fluidité. Ça va ailleurs que là où l'on allait. Quel plaisir si ripa dérivait de rivus, si c'était le ruissellement qui déterminait la rive! Le bord du ruisseau, de l'océan, se déplace avec lui. » Cf. J-F Lyotard, Dérive à partir de Marx et Freud, Union générale d'éditions 10/18, Paris 1973, p. 18.

La dérive a été pour lui une dérive hors de la critique; elle fut par elle-même la fin de la critique. La dérive marque l'importance du déplacement, son inconsistance par rapport à la pleine lumière de la raison. Il a inscrit, dans son livre Dérive à partir de Marx et Freud, en 1973, sa dérive en relation au marxisme radical. "La dérive a commencé pour moi au début des années 50 quand j'ai embarqué sur la nef de ces fous qui éditèrent la revue Socialisme ou barbarie et le journal Pouvoir ouvrier, et qui firent naufrage ou escale en 64-66 après quelques quinze ans de navigation hauturière. Comme c'est toujours le cas, notre divagation était toute sagesse: nous nous trouvâmes, chacun de son côté, à peu près de plain-pied dans le mouvement de 68, qui nous paraissait faire et dire en grand ce que nous avions esquissé par mots et actions en miniature et par prémonition, et qui inventa encore bien plus belles choses auxquelles nous n'avions pas pensé » Ibid, p. 11.
} 


\section{Grevisto}

ISSN n² 2447-4266

Vol. 4, n. 2, Abril-Junho. 2018

DOI: http://dx.doi.org/10.20873/uft.2447-4266.2018v4n2p973

nouvelle ascèse ? N'est pas cela que désigne ou appelle le mot de dérive? Et faut-il dire que ce nouveau travail implique de se déprendre du point de vue de classe? Le différend entre Lyotard et Souyri n'impliquera-t-il pas, pour le premier, un nouveau style de vie, à l'écart du radicalisme de classe ? Et dans quelle langue débattre de tout cela?

Pour Lyotard l'idiome du marxisme universel était décisif ; il semblait être l'enjeu même de son différend avec Souyri. Pourtant la complexité de ce différend a permis dégagé laissé un reste. Et ce reste, précise Lyotard, "c'était peut-être cela, la chose que mon différend même avec Souyri, et paradoxalement le retrait du marxisme pour moi, avait dégagée comme plus élémentairement politique que toute divergence, et dans laquelle les divergences prenaient corps. » (MPM, p. 114 et 115).

C'est cette chose qui est au cœur de la " genèse affectuelle » du concept de différend; en elle persiste toujours quelque chose du marxisme. Cela veut dire qu'il y avait dans le genre du discours marxiste, selon Lyotard, "quelque chose » qui échappait non seulement à la réfutation du marxisme, mais à sa propre décadence. Et ce qui y échappait à la décadence, à sa décrépitude

c'était tout d'abord l'affranchissement de la capacité critique, la réaffirmation que le point de vue de classe ne devait épargner aucun objet, et que la tâche principale des révolutionnaires était de détecter partout le différend, même là où il se cachait sous de simples divergences. (MPM, p. 118)

C'est cette «chose » même, qui du marxisme n'était pas finie, c'est elle qui conservait toujours son autorité sur le vouloir et sur le penser. Cette chose qui lui a donné à penser, c'est précisément le concept de différend qui viendrait 


\section{Obevisto}

ISSN n² 2447-4266

Vol. 4, n. 2, Abril-Junho. 2018

DOI: http://dx.doi.org/10.20873/uft.2447-4266.2018v4n2p973

la nommé, différend que Marx lui-même avait déjà mis à l'œuvre dans son analyse du Capital:

Cette chose que j'appelle ici le différend porte dans la «tradition» marxiste le nom "bien connu» qui donne lieu à beaucoup de méprises, celui de pratique ou " praxis », le nom que par excellence la pensée théorique mésinterprète. (...) S'il y a une pratique de classe, alors que le concept ne donne pas lieu à pratique, c'est que l'universalité n'est pas exprimable en mots, sauf unilatéralement. Les rôles des protagonistes de l'histoire ne se jouent pas dans un seul et même genre de discours. Le capital qui se donne pour la langue universelle est par là même ce qui révèle la multiplicité d'idiomes intranscriptibles. Entre ceux-ci et la loi de la valeur, le différend ne peut pas se résoudre par la spéculation ou dans l'éthique, il doit l'être dans la "pratique», que Marx appelait pratique-critique, dans un combat incertain contre la partie qui se donne pour le juge. (MPM. p. 116 et 117).

Alors, le différend est le nom de la «praxis » marxiste, c'est-à-dire la pratique-critique; il dévoile l'illusion et donc le tort d'un discours que prétend être universel. Mais dans la mesure où le marxisme prétendait pouvoir exprimer sans résidu toutes les positions antagonistes, il rencontrait lui-même sa propre contradiction interne. Marx avait montré qu'il y avait au moins deux idiomes en contradiction cachés dans la langue du capital.

Si Le Capital avait été la critique, ou une critique, de l'économie politique, c'était pour avoir fait entendre le différend là où il était caché sous l'harmonie, ou du moins sous l'universel. Marx avait montré qu'il y avait au moins deux idiomes ou deux genres cachés dans la langue universelle du capital, l'AMA' [argent-marchandise-plus d'argent], que parlait le capitaliste, et le MAM [marchandise-argentmarchandise] que parlait le salarié. Le locuteur de l'un entendait fort bien le locuteur de l'autre, et les deux idiomes étaient traduisibles l'un dans l'autre, mais il y avait entre eux une différence qui faisait qu'en 


\section{Gevisto
Observatório}

ISSN n² 2447-4266

Vol. 4, n. 2, Abril-Junho. 2018

DOI: http://dx.doi.org/10.20873/uft.2447-4266.2018v4n2p973

transcrivant une situation, une expérience, un référent quelconque exprimé par l'un dans l'idiome de l'autre, ce référent devenait méconnaissable pour le premier, et le résultat de la transcription incommensurable avec l'expression initiale. (MPM, p. 115)

En somme, l'analogie devient évidente entre le différend dans lesquelles est pris le salarie parlant la langue du capital, et le différend dans lequel est situé le militant dissident tant qu'il n'a que la langue du marxisme pour dire son soupçon.

\section{IV}

\section{Le Différend}

Dans cet exemple, autobiographique à bien des égards, nous trouvons au cœur de la pensée philosophique de Lyotard. Nous pouvons lire la problématique du marxisme comme "forme de savoir », dit de "spiritualités », dans le texte de Lyotard "Mémorial pour un marxisme: A Pierre Souyri». Foucault montre l'importance de cette « forme de savoir » pour la formation ou transformation du sujet, dans le cas de la vie militante ou révolutionnaire.

Foucault marque en même temps une réserve à l'égard de cette forme de savoir marxiste (tout autant que psychanalytique, d'ailleurs). C'est que dans la mesure où elle se référerait à une médiation institutionnelle extérieure au travail sur le rapport entre vérité et sujet, elle induirait un oubli de ceux-ci.

L'idée d'une position de classe, d'effet de parti, l'appartenance à un groupe, l'appartenance à une école, l'initiation, la formation de l'analyste, etc., tout ceci nous renvoie bien à ces questions de la condition de la formation du sujet pour l'accès à vérité, mais on les pense en termes sociaux, en termes d'organisation. »Et Foucault d'ajouter: «Et en même temps d'ailleurs, le prix payé pour transposer, 


\section{Qrevisto}

ISSN n² 2447-4266

Vol. 4, n. 2, Abril-Junho. 2018

DOI: http://dx.doi.org/10.20873/uft.2447-4266.2018v4n2p973

pour rabattre ces questions "vérité et sujet » sur des problèmes d'appartenance (à un groupe, à une école, à un parti, à une classe, etc.), le prix payé, ça a été bien entendu l'oubli de la question des rapports entre vérité et sujet. ${ }^{11}$

Lyotard a connu «le prix à payer » pour avoir tâché de reprendre à nouveau frais les rapports entre vérité et sujet ; c'est-à-dire, pour se questionner sur lui-même, pour faire un travail sur soi-même, exigent de lui une transformation de sa manière de penser et de vivre. La vérité ne se donne pas d'emblée, il faut «payer le prix » pour y accéder, s'adonner à des exercices spirituels à fin de se rendre capable de vérité. ${ }^{12}$. Ce prix a consisté à faire son deuil de la perte de l'amitié et de la légitimation du discours marxiste, après sa découverte que le discours marxiste ne peut pas exprimer les changements du monde contemporain (cf. MPM, p.98). Ou comme le dit encore Lyotard: "Si en vérité l'enjeu [de la vraie vie] n'était pas la suppression des rapports d'exploitation, l'échec était seulement celui de la pensé qui prétendait l'inverse. » (MPM, p.102).

C'est son différend avec Souyri qui a rendu possible sa dérive et sa recherche des nouveaux rapports entre vérité et sujet; cela veut dire que son différend a exigé une nouvelle transformation de soi, ouvrent sur des nouvelles manières de penser.

Or, si nous prenons l'exemple de Marx, celui du capital par rapport au travailleur, et si nous posons aussi notre exemple du militant à l'égard du discours marxiste (cas de Lyotard), nous pouvons alors construire une

\footnotetext{
${ }^{11}$ Cf. Foucault, L'Herméneutique du sujet, leçon du 6 janvier 1982, p. 31.

${ }^{12}$ D'ailleurs comme Lyotard le définira par la suite, la vérité apparaît toujours là où on n'attendait pas, il faut bien donc une nouvelle ascèse pour l'accueillir ; Cf. Dérive dans Marx et Freud.
} 


\section{Grevisto}

ISSN n² 2447-4266

Vol. 4, n. 2, Abril-Junho. 2018

DOI: http://dx.doi.org/10.20873/uft.2447-4266.2018v4n2p973

importante analogie concernant le concept de différend développé par Lyotard. Dans les deux exemples, l'une des parties a la prétention d'être un langage universel, donc prétention d'être partie et juge à la fois. Dans le premier cas, c'est le capital ; dans le deuxième, c'est le discours marxiste. Or, dit Lyotard :

\footnotetext{
Le marxisme est alors l'intelligence critique de la pratique du déchirement, dans les deux sens: il déclare le déchirement «audehors », dans la réalité historique; le déchirement « au-dedans » de lui, comme différend, empêche cette déclaration d'être universellement vraie une fois pour toutes. (MPM, p. 134)
}

Le premier cas (du Capital) est celui qui a élucidé, qui contenait en germe le concept de différend; le deuxième (le cas du militant) est précisément celui qui a amené le militant Lyotard à faire un nouveau et sévère travail sur luimême, lequel a rendu possible un changement dans sa pensée et dans vie, qui a ouvert le champ à une vita nuova, à « sa philosophie », celle du Différend.

\section{OUVRAGES CONSULTES}

FouCAULT, M., François Ewald, Alessandro Fontana, et Frédéric Gros. L'herméneutique du sujet: cours au Collège de France, 1981-1982. Hautes études. Paris : Gallimard Seuil, 2001.

- Frédéric Gros, François Ewald et Alessandro Fontana. Le Courage de la Vérité. Le gouvernement de soi et des autres II. Cours au collège de France. 1984. Hautes études. Paris : Gallimard Seuil, 2009. 


\section{revisto}

ISSN n²447-4266

Vol. 4, n. 2, Abril-Junho. 2018

DOI: http://dx.doi.org/10.20873/uft.2447-4266.2018v4n2p973

- Henri-Paul Fruchaud, et Daniele Lorenzini. L'origine de l'herméneutique de soi: conférences prononcées à Dartmouth College, 1980. Philosophie du présent. Paris : J. Vrin, 2013.

—. Subjectivité et vérité: cours au Collège de France, 1980-1981. Hautes études. Paris : EHESS Gallimard Seuil, 2014.

LYOTARD, J-F. Dérive à partir de Marx et Freud. 10-18 754. Paris: Union générale d'éditions, 1973.

—. Instructions païennes. Débats. Paris : Galilée, 1977.

—. Le différend. Collection Critique. Paris : Les éd. de Minuit, 1983.

—. Pérégrinations : loi, forme, événement. Débats. Paris : Galilée, 1990.

-. Rudiments païens: genre dissertatif. Continents philosophiques 6. Paris : Klincksieck, 2011.

Prado, P. «Le sentiment du différend ou la critique de raison communicationnelle » in Prado, P., Cany, B., Poulain, J. (org.) Passages de JeanFrançois Lyotard : rencontre internationale, Paris, 14-17 octobre 2009. Paris : Hermann, 2011.

—. "La dette d'affect », in L'exercice du différend - J.-F. Lyotard, Actes du Colloque international org. par D. Lyotard, J.-Cl. Milner et G. Sfez, Paris : Collège international de philosophie / PUF, 2001. 